\title{
A Survey for Low Surface Brightness Galaxies using Tech Pan Films
}

\author{
Jean Marc Schwartzenberg and Steven Phillipps
}

Department of Physics and Astronomy, University of Wales College of Cardiff, P.O. Box 913, Cardiff CF2 3YB, U.K.

\author{
Quentin A. Parker
}

UKST, Anglo-Australian Observatory, Siding Spring Mountain, Coonabarabran, NSW 2357, Australia

\begin{abstract}
We report on a new survey for low surface brightness galaxies using Kodak Tech Pan 4415 film on the UKST, and demonstrate the advantages of this film over conventional IIIa plates when searching for faint, extended objects. Recent experiments with films of the Virgo cluster have shown that the noise per pixel in deep exposures is only $0.7 \%$ of sky, enabling known objects to be traced out to an isophote around $27 R \mu$. In addition, a preliminary survey has revealed several new galaxies with central surface brightnesses around $24 R \mu$. With the digital coaddition of several films, we expect to be able to measure objects out to the $28 R \mu$ isophote and detect galaxies with central surface brightnesses as faint as $\sim 25 R \mu$, i.e. below $3 \%$ of sky.
\end{abstract}

\section{Introduction}

Searches for faint, low surface brightness galaxies (LSBGs) have in the past been limited either by detector area or detector efficiency. Conventional UKST plates cover large areas but have inherent signal-to-noise (S/N) limitations, while CCDs reach deep limits but sample much smaller areas of the sky in a given amount of time. A new, very promising way to overcome the S/N limitations of Schmidt plates while retaining the same area coverage is the use of Kodak Tech Pan 4415 (hereafter referred to as " 4415 ") film as a substitute for conventional IIIa plates.

We are now benefiting from the substantial advantages of 4415 film to undertake a survey of LSBGs in the Virgo cluster. Besides reaching fainter surface brightnesses than previous LSBG surveys (e.g. Binggeli et al. 1985; Davies et al. 1988; Impey et al. 1988), our higher S/N will allow the inclusion of objects with considerably smaller angular diameters, thus contributing a significant new population of LSBGs to that currently known.

\section{The use of Tech Pan films on the UKST}

Despite its relatively recent appearance in professional astronomy, 4415 film now constitutes a considerable fraction of UKST output. As its technical details have been described elsewhere (see Parker, these proceedings), we will concentrate here on its advantages for the detection and photometry of faint galaxies. 
The fine grain, high quantum efficiency and improved sensitivity of Tech Pan film over traditional IIIa emulsions mean that fainter limiting isophotes can be attained in a typical 75 minute, sky-limited exposure. A detailed photometric study of five known Virgo cluster galaxies from COSMOS scans of 4415 film (Phillipps \& Parker 1993) has shown that typical pixel-to-pixel variations on $1^{\prime \prime}$ scales are as low as $0.7 \%$, i.e. a factor of 3 improvement in uniformity over the corresponding IIIa-F emulsion. This very low sky noise means that we can perform surface photometry to a limiting isophote of $27.0 R \mu$, equivalent to only $0.25 \%$ of the sky brightness. 4415 film therefore offers a gain of about 1.5 magnitudes over IIIa-F emulsion in this respect.

\section{The Virgo film survey}

Nearby clusters such as Virgo offer the ideal environment in which to search for LSBGs. It is now known that LSBGs dominate cluster numbers (Turner et al. 1993), and can therefore represent a significant fraction of a cluster's total baryon content. Furthermore, preliminary results from a CCD field survey (Schwartzenberg et al., in preparation) show that very few apparently large LSBGs are present in random field areas, and thus a cluster environment must be sampled if we are to find large numbers of LSBGs.

Our LSBG survey is based on COSMOS digital scans of eight independent 4415 exposures ( 75 minutes each) of a $5^{\circ} \times 5^{\circ}$ area of the Virgo Cluster. A preliminary visual search in a 0.5 square degree subset of this field has revealed previously uncatalogued LSBGs with central surface brightnesses $\mu_{o}(R) \simeq 23.5-$ 24.0 and scale lengths $4-5$ arcsec. An image and profile of one of these LSBGs are shown in Fig. 1. We have also identified, with remarkable ease, some of the faintest LSBGs included in the study of Impey et al. (1988).

The search over the whole 25 square degree area will be carried out in an automated fashion, using connected pixel detection algorithms which should enable the detection of LSBGs down to very small scale lengths, $\alpha \simeq 33^{\prime \prime}$, corresponding to about $320 \mathrm{pc}$ at Virgo (assuming $H_{0}=50 \mathrm{kms}^{-1} \mathrm{Mpc}^{-1}$ ). This linear scale length is comparable to that of the dwarf spheroidals in the Local Group (Caldwell et al. 1992). We will therefore be sampling not only to fainter limits when compared to previous Virgo surveys (Binggeli et al. 1985, Impey et al. 1988), but also to smaller angular sizes, thus allowing for the inclusion of physically smaller LSBGs, which are thought to be most common (see below).

\section{Conclusions/future prospects}

Our discovery of two new LSBGs in the Virgo cluster has highlighted the potential of Tech Pan films for the detection and photometry of faint galaxies. Further gains can be expected from the coaddition of all our eight individual exposures, which should push the detection limit further by 1.1 magnitude. Artefacts present on individual frames can also be removed by means of medianstacking. As the number of LSBGs increases rapidly with decreasing scale length $\left(n(\alpha) \propto \alpha^{-2}\right.$, Irwin et al. 1990), the small angular size limit of our survey, combined with its fainter surface brightness limit, will allow us to investigate the contribution of LSBGs to the cluster luminosity function, as well as their covering factor and spatial distribution. 

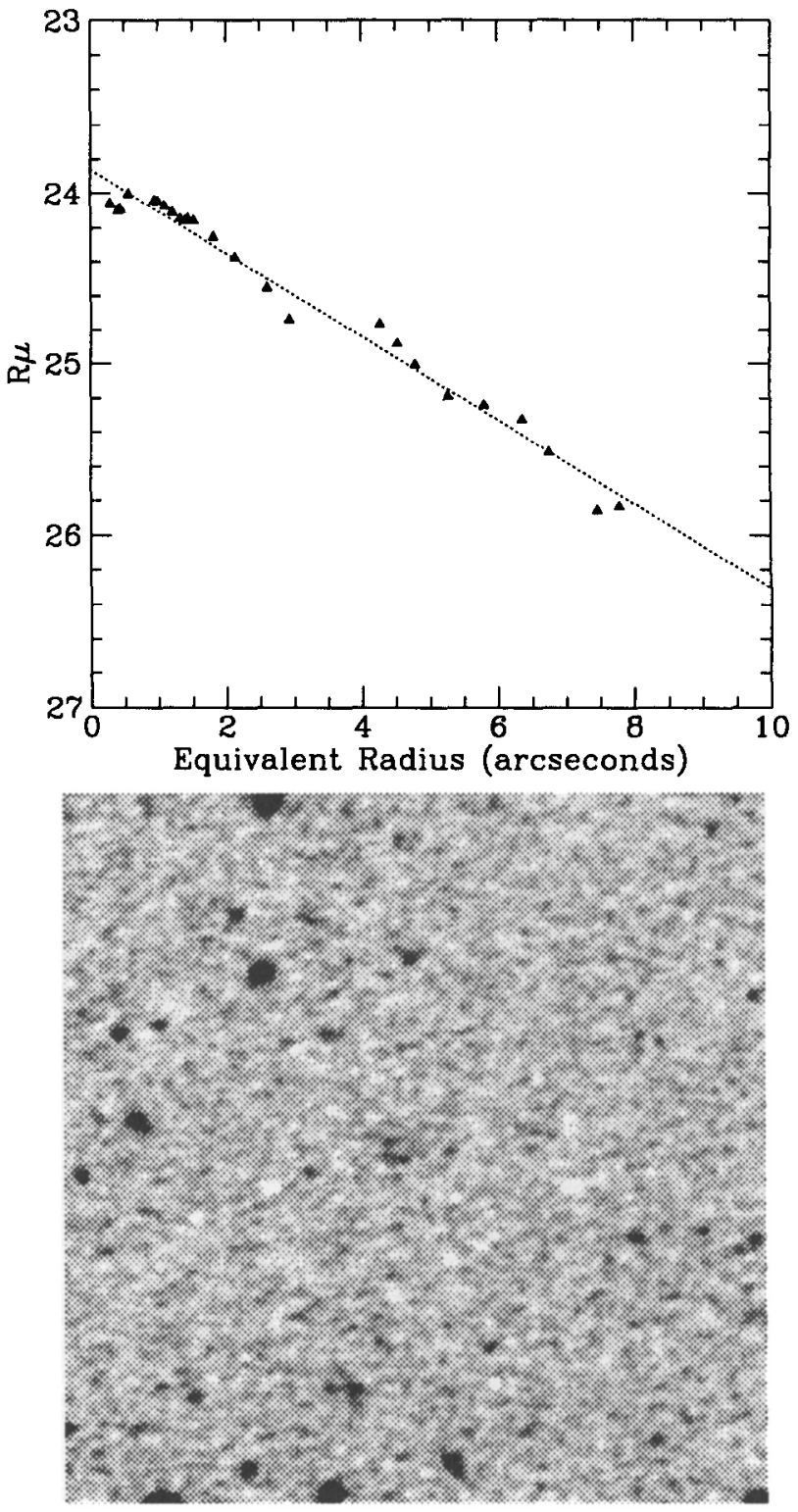

Figure 1. (a) Surface brightness profile and (b) grey-scale image of LSBG at $12^{h} 29^{m} 59^{s}, 13^{\circ} 31^{\prime} 42^{\prime \prime}(1950.0)$. The LSBG is the diffuse object at the centre of the image. North is at the top, east is at the left. The image is $3 \times 3$ arcminutes. The dotted line in $1(\mathrm{a})$ represents the best fit to the galaxy's profile. 


\section{References}

Binggeli B., Sandage A. \& Tammann G. A., 1985, AJ, 90, 1681

Caldwell N., Armandroff T. E., Seitzer P. \& Da Costa G. S., 1992, AJ, 103, 840

Davies J. I., Phillipps S., Cawson M. G. M., Disney M. J. \& Kibblewhite E. J., 1988, MNRAS, 232, 239

Impey C. D., Bothun G. D. \& Malin D. F., 1988, ApJ, 330, 634

Irwin M. J., Davies J. I., Disney M. J. \& Phillipps S., 1990, MNRAS, 245, 289

Phillipps S. \& Parker Q. A., 1993, MNRAS, 265, 385

Turner J. A., Phillipps S., Davies J. I. \& Disney M. J., 1993, MNRAS, 261, 39

\section{Discussion}

Armandroff: You cited an area advantage of 400:1 of plates over CCD imagers. We have heard about two Schmidt CCD systems at this meeting, with a field of one square degree (Burrell Schmidt and Beiging Schmidt). This would seem to suggest a smaller advantage of 36:1. One should also remember the quantum efficiency advantage of CCDs, and the fact that potential future imagers with areas up to 10 square degrees are under discussion.

Schwartzenberg: The ratio of 400:1 refers to a $1024 \times 1024$ CCD operating at $f / 1$ on a conventional telescope. While there is no doubt that CCDs on Schmidts will greatly reduce this ratio, one should bear in mind the extra amount of spatial information contained in Tech Pan films, which have an inherent resolution of only $3 \mu \mathrm{m}$, well below the current size of CCD pxiels.

Drinwater: Do you have any measure on whether the smaller galaxies you have detected are cluster members or background objects?

Schwartzenberg: A separate survey for low surface brightness galaxies in the field (in progress) should provide a direct measurement of the amount of backgound contamination. It is not clear whether cluster membership based on morphology, as successfully employed by Binggeli, Sandage \& Tammann (1985), will be possible at such small angular sizes.

Cannon: I was very interested in the internal structure which you see in these faint galaxies. Can you confirm its reality by looking at separate films, or subsets of the data? The Galactic dwarf spheroidals are notable for being rather smooth and featureless.

Schwartzenberg: Indeed, we have checked the reality of our objects and their internal structure by comparing individual films. The discrepancy with local dwarf spheroidals might therefore be due to the effects of different environments on low surface brightness galaxies. 